\title{
Structure of the phonon vacuum state
}

\author{
S. Mishev* \\ Joint Institute for Nuclear Research, 6 Joliot-Curie str., Dubna 141980, Russid
}

(Dated: September 3, 2018)

\begin{abstract}
The action of the long-range residual force on the expectation value of observables in the nuclear ground states is evaluated by finding optimal values for the coefficients of the canonical transformation which connects the phonon vacuum state with the (quasi-)particle ground-state. After estimating the improvements over the predictions of the independent-particle approximation we compare the ground-state wave functions, obtained using the presented approach, with those, obtained using the conventional random phase approximation (RPA) and its extended version. The problem with overbinding of the nuclear ground state calculated using the RPA is shown to be removed if one sticks to the prescriptions of the present approach. The reason being that the latter conforms to the original variational formulation. Calculations are performed within the two-level Lipkin-MeshkovGlick model in which we present results for the ground and first excited state energies as well as for the ground-state particle occupation numbers.

PACS numbers: 21.60.Jz, 21.60.Ev, 21.60.Cs
\end{abstract}

\section{INTRODUCTION}

Approximating the correlated ground state of a manybody quantum system has been receiving considerable attention since the early days of nuclear structure physics [1] and degenerate electron gas theory [2] and still represents a formidable challenge. This is an arduous task within the "beyond the mean-field" theories because of the action of the residual interaction which brings particle-hole admixtures into the ground states. In the present paper we focus on the effects of long-range part of this interaction. Unambiguously attributable to the latter is the quadrupole correlation energy, which, as shown by the findings in Ref. [3], is considerable and varies between $100 \mathrm{keV}$ and $5.5 \mathrm{MeV}$ in different nuclei which makes the perturbative treatment unsuitable. The short-range residual forces, on the other hand, compete with the long-range ones in dominating the ground-state shapes formation [4, 5]. As a result of this competition, in the beginning and the end of major shells the nucleons are paired, giving rise to spherical shape, while in the middle of the shell the nucleons are paired-off and they align to the field generating forces thus contributing to deformation. We conjecture that the present study can serve as a foundation to investigate the mechanism of the transition between these two regimes and in particular on the pairs decoupling process. The process that we surmise was concocted in Ref. [6] and essentially implies that the long-range force breaks nucleon pairs which may further recouple due to the pairing force.

We approximate the nuclear ground-state wave function with the phonon operators [7] vacuum state. A general form of the phonon vacuum was proposed by Sorensen [8] and later Goswami and Pal [10] estimated

\footnotetext{
*mishev@theor.jinr.ru

$\uparrow$ On leave from Institute for Nuclear Research and Nuclear Energy, Bulgarian Academy of Sciences
}

explicitly the correlation coefficients of the $2 p-2 h$ admixtures into the BCS wave function [11] relating them to the forward and backward phonon amplitudes. The relation they obtained turned out to be also valid for higher order correlations [12] in the random phase approximation (RPA) 1]. Being a small amplitude limit of the timedependent Hartree-Fock approximation, however, RPA is known to be able to account only for small correlation effects. Since in open-shell nuclei the backward phonon amplitudes are by no means small, RPA is becoming questionable in describing the low-energy states of such nuclei. On a quest to construct a microscopic global theory for the nuclear binding energies it was shown in Refs. 19, 20] that the RPA is a useful method around spherical and well deformed nuclei but falls short in describing nuclei from the transitional region. This problem was addressed by Hara [6] and later on by Jolos and Rybarska 14], who proposed an improvement over the RPA, referred to as extended RPA(ERPA), based on the Pauli blocking principle which plays a progressively important role with the increasing number of the valence nucleons. This extension has proven to be in better accord with the experimental data as demonstrated in a number of papers as for example in Refs. 15, 16]. Although its superiority over the standard RPA is undeniable, the variational character of the theory is violated as the ground state is found to be overbound. The strong argument that the variational property of a theory insures a converging succession of approximations to the exact solution fostered the formulation of a elaborate formalism, called self-consistent RPA [17], which as in the conventional particle-hole theory, allowed to take into account the nucleon correlations without explicitly constructing the ground-state wave function. In Ref. [18] within the schematic two-level Lipkin-Meshkov-Glick(LMG) model 29] it is found that the self-consistent theory allows one to go beyond the point of RPA collapse, but near this point the wave function of the first excited state within this approximation is found to be almost orthogonal to 
the exact one. This behavior is due to the higher order particle-hole admixtures contributing to the structure of the first excited state.

We note that beside the aforementioned amendments to the standard RPA, there exist a number of other important developments on this subject as, for example, those in Refs. [21 27].

In the present paper we keep using the explicit form of the fermionic many-body vacuum [8] but depart from varying the excited-state wave function. On the contrary we use the correlation coefficients as parameters which we fix by optimizing the ground-state trial wave function using a variational procedure. The excited states corresponding to such vacuum state are of $1 p-1 h$ type. This approach also benefits from the findings in Ref. [28] where it was shown that this class of wave functions is a vacuum for a generalized phonon operator, adding to the standard one specific two-body operators correcting for the Pauli principle. By way of example, using the two-level LMG model, they showed that the additional terms improve the convergence substantially. In this way the phonon vacuum state absorbs additional correlations effects than the ones obtained using the equations-of-motion method [4] for the standard phonon operator.

The paper proceeds as follows. In Section II we outline the problem and summarize the main obstacles towards the exact solution. Basic equations of several approximate methods including the RPA, ERPA and the explicit variation of the phonon vacuum state along with the exact solution within the LMG model are derived in Section III. A comparison between them is established on the basis of the ground and the first excited state energies as well as the on ground-state particle occupation numbers in Section IV. Summary and outlook is given in Section V.

\section{FORMULATION}

Formally a wave function which contains admixtures into the independent-quasiparticle wave function and is a vacuum for the phonon operators [7]

$Q_{\lambda \mu i}=\frac{1}{2} \sum_{11^{\prime}}\left[\psi_{11^{\prime}}^{\lambda i} A\left(11^{\prime} \mid \lambda \mu\right)-(-1)^{\lambda-\mu} \varphi_{11^{\prime}}^{\lambda i} A^{\dagger}\left(11^{\prime} \mid \lambda-\mu\right)\right]$

can be expressed as [8]

$$
\left.|\rangle=N_{0} \cdot e^{\hat{S}}\right\rangle
$$

with

$\hat{S}=-\frac{1}{2} \sum_{12 ; \lambda \mu} S_{\lambda}\left(11^{\prime} ; 22^{\prime}\right)(-)^{\lambda-\mu} A^{\dagger}\left(11^{\prime} \mid \lambda \mu\right) A^{\dagger}\left(22^{\prime} \mid \lambda-\mu\right)$.
Here and below an independent shell-model state $\left\{N_{1}, l_{1}, j_{1}, m_{1}\right\}$ is abbreviated as 1 .

The coefficients $S_{\lambda}\left(11^{\prime} ; 22^{\prime}\right)$ are referred to as correlation coefficients and denote the amplitudes for the presence of zero, four, eight, ... quasiparticles in the ground state due to the virtual vibrations. These present a primary source of structure information for the ground states and make up a major part of our present research. The correlated and uncorrelated ground states are denoted as |\rangle and $\rangle$ correspondingly. The two-quasiparticle creation operator $A^{\dagger}(12 ; \lambda \mu)$ reads

$$
A^{\dagger}(12 ; \lambda \mu)=\sum_{m_{1} m_{2}}\left\langle j_{1} m_{1} j_{2} m_{2} \mid \lambda \mu\right\rangle \alpha_{j_{1} m_{1}}^{\dagger} \alpha_{j_{2} m_{2}}^{\dagger}
$$

where $\alpha_{j m}^{\dagger}$ denotes the quasiparticle creation operator.

In the following we shall also need the quasiparticle scattering operator

$$
B(12 ; \lambda \mu)=\sum_{m_{1} m_{2}}(-)^{j_{2}+m_{2}}\left\langle j_{1} m_{1} j_{2} m_{2} \mid \lambda \mu\right\rangle \alpha_{j_{1} m_{1}}^{\dagger} \alpha_{j_{2}-m_{2}} .
$$

Details on the quasiparticle-phonon nomenclature, which we follow in this paper, can be found in Ref. 7].

Using the ERPA, the correlation coefficients are found to satisfy the equations [6, 8]

$$
\varphi_{11^{\prime}}^{\lambda i}=\sum_{22^{\prime}}\left(1-\rho_{22^{\prime}}\right) S_{\lambda}\left(11^{\prime} \mid 22^{\prime}\right) \psi_{22^{\prime}}^{\lambda i}
$$

where $\rho_{1}$ is the quasiparticle occupation density on the level 1.

In Eq.(2) $N_{0}$ is a normalization factor which in physical terms is the overlap between the independent-particle and the correlated wave functions. It is found to be

$$
N_{0}^{2}=\frac{1}{\left\langle e^{\left(S^{\dagger}+S\right)}\right\rangle}
$$

In RPA, suggesting small correlations so that higher order terms contribute relatively little, this constant is approximated as [12, 13]:

$$
N_{0}^{2} \approx \frac{1}{e^{\frac{1}{2} \sum_{11^{\prime} 22^{\prime} ; \lambda} \pi_{\lambda}^{2} S_{\lambda}^{2}\left(11^{\prime} \mid 22^{\prime}\right)} .}
$$

Here and below we use the shortcut notation $\pi_{\lambda}=$ $\sqrt{2 \lambda+1}$, with $\pi_{\lambda \lambda^{\prime}}=\pi_{\lambda} \pi_{\lambda^{\prime}}$. An explicit solution to the system (6) was obtained by Hara [6].

Changing the frame of mind we shall try to obtain the correlation coefficients by explicitly varying the wave function |\rangle in the functional

$$
\delta\langle|H|\rangle=0
$$

, with $S_{\lambda}\left(11^{\prime} ; 22^{\prime}\right)$ being variational parameters, i.e. we shall try to solve the equation 
$\delta\left(N_{0}^{2}\left\langle e^{S^{\dagger}} H e^{S}\right\rangle\right) \equiv\left\langle e^{S^{\dagger}} H e^{S}\right\rangle\left(\delta N_{0}^{2}\right)+N_{0}^{2}\left(\delta\left\langle e^{S^{\dagger}} H e^{S}\right\rangle\right)=0$

with respect to $S_{\lambda}\left(11^{\prime} ; 22^{\prime}\right)$. Assuming that states with more than four quasiparticles are less probable to be excited due to the pairing gap [1], i.e. the configuration space for the ground state is restricted to four quasiparticle admixtures only, the quantities that need to be evaluated are presented in the following expression

$$
N_{0}^{2}\left\langle e^{S^{\dagger}} H e^{S}\right\rangle \approx \frac{\langle H\rangle+2\langle H S\rangle+\left\langle S^{\dagger} H S\right\rangle}{1+\frac{1}{2}\left\langle S^{\dagger} S\right\rangle} .
$$

The validity of this assumption is examined in Sec. IV using a simplified setup provided by the LMG model, where it is shown that it holds true at strengths weaker than or in the vicinity of the RPA point of collapse and is incorrect in the strong interaction regime where higher order correlations start to play an important role.

A realistic Hamiltonian in quasiparticle representation which accounts for the mean-field, monopole pairing and the isoscalar, multipole-multipole long-range part of the residual interaction has the following form

$$
H=H_{q p}+H_{r e s}
$$

where

$$
H_{q p}=\sum_{j m} \varepsilon_{j} \alpha_{j m}^{\dagger} \alpha_{j m}
$$

Expressions for the quantities in Eqs. (17) and (11) using the above Hamiltonian, in the case of one-nucleon species, are given in App. A There it is shown that under the limiting conditions listed in Sec. ПIIA these expressions coincide with the ones obtained within the LMG model with $2 p-2 h$ admixtures into the ground state. The numerical solution of Eq. (10) is left out as a subject for a future study.

A common technique, which allows to mimic the dynamics govern by the Hamiltonian (15), from one side, and provides a tractable way of evaluating these quantities, from the other, is to have recourse to exactly solvable models which, in our case, would ideally incorporate pairing and quadrupole terms. This however proves impossible due to the fact that these two interactions are associated with incompatible symmetry groups [9]. In this paper we used the simplistic and widely used LMG model as a testbed for proving the correctness of our idea.

\section{SOLUTION WITHIN THE LIPKIN-MESHKOV-GLICK MODEL}

\section{A. LMG model basics}

In order to access the utility of different approaches and to prove the usefulness of the proposed scheme we limit the configuration space and simplify the internucleon interaction to monopole-monopole one as suggested by Lipkin, Meshkov and Glick [29, 30]. This setting permits comparisons between the rates of convergence of different approximation methods, including the hereby described, to the exact solution.

In this model the interaction of $N$ particles on 2 quantum levels is presented by the following Hamiltonian

$$
H=H_{0}+V ; H_{0}=\varepsilon J_{0} ; V=\frac{G}{2}\left(J_{+}+J_{-}\right)^{2},
$$

where

$$
\begin{aligned}
& J_{+}=\sum_{i} a_{1 i}^{\dagger} a_{-1 i}, \\
& J_{-}=\sum_{i} a_{-1 i}^{\dagger} a_{1 i}, \\
& J_{0}=\frac{1}{2} \sum_{i}\left(a_{1 i}^{\dagger} a_{1 i}-a_{-1 i}^{\dagger} a_{-1 i}\right)
\end{aligned}
$$

are analogous to the raising, lowering and angular momentum' $z$-component of the quasi-spin algebra respectively, $a^{\dagger}$ represents the particle creation operator, the suffix \pm 1 denotes the upper or lower level, $\varepsilon$ is the energy gap between the two levels and $G$ is the interaction strength. 
We shall also make use of the operators

$s_{n}^{+}=a_{1 n}^{\dagger} a_{-1 n} ; s_{n}^{-}=a_{-1 n}^{\dagger} a_{1 n} ; s_{n}^{0}=\frac{1}{2}\left(a_{1 n}^{\dagger} a_{1 n}-a_{-1 n}^{\dagger} a_{-1 n}\right)$.

The Hamiltonian (15) can be considered as a specialization (up to a constant term) of the more general one (14) under the following simplifications:

- pairing is switched off;

- the number of levels is reduced to only two $\{-1,1\}$, each with a particle capacity of $N$;

- the monopole-monopole part of the interaction is only considered;

- the terms in the Hamiltonian (14), quadratic with respect to operator $B$ (Eq.(5) ), are neglected.

The operators (16) can be expressed by the ones defined in Eqs. (44) and (5) in the following way

$$
\begin{gathered}
J_{+}=-\sqrt{N} A^{\dagger}, \quad J_{-}=-\sqrt{N} A, \\
J_{0}=\frac{1}{2} \sqrt{N}\left(B_{+1}+B_{-1}-\sqrt{N}\right),
\end{gathered}
$$

where

$$
\begin{aligned}
& A^{\dagger}=A^{\dagger}((-1)(+1) ; 00), \\
& B_{+1}=B((+1)(+1) ; 00), \\
& B_{-1}=B((-1)(-1) ; 00) .
\end{aligned}
$$

The interaction strengths in Eqs. (15) and (14) are related as

$$
G=-\frac{\kappa_{0}^{0}}{N}
$$

\section{B. Exact solution}

The exact solution of the many-body problem is obtained as a superposition of the (normalized) states $|n\rangle$ with $0,1,2 \ldots, N$ particles on the upper level $(|0\rangle \equiv\rangle)$ :

$$
|\Psi\rangle=\sum_{n} c_{n}|n\rangle
$$

The weights $c_{n}$ are readily obtained by solving the eigenvalue problem

$$
\sum_{n^{\prime}}\left\langle n|H| n^{\prime}\right\rangle c_{n^{\prime}}=E c_{n}
$$

The non-zero elements of the matrix on the left-hand side of the above equation evaluate to

$$
\begin{aligned}
& \langle n|H| n\rangle= \\
& =\left(-\frac{N}{2}+n\right) \varepsilon+G\left(-\frac{N}{2}+n+(n+1)(N-n)\right)
\end{aligned}
$$

$$
\langle n|H| n+2\rangle=\frac{G}{2} \sqrt{n(n-1)(N+2-n)(N+1-n)} .
$$

For the ground-state total energy we then obtain

$$
E=\sum_{n}\left(c_{n}^{(0)}\right)^{2}\langle n|H| n\rangle+2 \sum_{n} c_{n}^{(0)} c_{n+2}^{(0)}\langle n|H| n+2\rangle
$$

We shall further present the solutions for excited states, containing only one particle on the upper level and one hole on the lower one, i.e.

$$
\left.|1 p 1 h\rangle_{m}=\left(\sum_{i}\left(\psi_{i}^{(m)} s_{i}^{+}-\varphi_{i}^{(m)} s_{i}^{-}\right)\right)\right\rangle
$$

\section{RPA}

Employing the RPA, i.e.

$$
\left\langle\left|s_{i}^{-}, s_{i^{\prime}}^{+}\right|\right\rangle=\delta_{i i^{\prime}}
$$

one obtains the well-known equation for the excitation energies and the forward and backward amplitudes:

$$
\begin{aligned}
& \left(\begin{array}{cc}
\delta_{i i^{\prime}}(\varepsilon-G)+G & -G\left(1-\delta_{i i^{\prime}}\right) \\
-G\left(1-\delta_{i i^{\prime}}\right) & \delta_{i i^{\prime}}(\varepsilon-G)+G
\end{array}\right)\left(\begin{array}{l}
\psi_{i^{\prime}}^{(m)} \\
\varphi_{i^{\prime}}^{(m)}
\end{array}\right)= \\
& =\omega\left(\begin{array}{cc}
\delta_{i i^{\prime}} & 0 \\
0 & -\delta_{i i^{\prime}}
\end{array}\right)\left(\begin{array}{l}
\psi_{i^{\prime}}^{(m)} \\
\varphi_{i^{\prime}}^{(m)}
\end{array}\right),
\end{aligned}
$$

which together with the normalization of the wave functions (30) yields a collective solution

$$
\begin{aligned}
& \psi=\frac{1}{\sqrt{N}} \frac{1+\chi / 2+\omega_{0} / \varepsilon}{\sqrt{\left(1+\frac{\omega_{0}}{\varepsilon}\right)\left(1+\chi+\frac{\omega_{0}}{\varepsilon}\right)}}, \\
& \varphi=\frac{1}{\sqrt{N}} \frac{\chi / 2}{\sqrt{\left(1+\frac{\omega_{0}}{\varepsilon}\right)\left(1+\chi+\frac{\omega_{0}}{\varepsilon}\right)}},
\end{aligned}
$$


where

$$
\chi=\frac{2 G(N-1)}{\varepsilon}, \omega_{0}=\varepsilon \sqrt{1+\chi} .
$$

Since the phonon amplitudes are independent of the particle-hole pair $i$ which they refer to due to the symmetry of the model and we are interested in the collective solution only the wave function (30) can be rewritten in the more compact form which we shall further use

$$
\left.|1 p 1 h\rangle=\left(\psi J_{+}-\varphi J_{-}\right)\right\rangle .
$$

The particle occupation of the lower LMG level is easy obtained as

$$
\rho=1-\varphi^{2} .
$$

\section{ERPA}

The condition (31) disregards some aspects of the nature of the excited states (30), in particular the fact that the number of particle-hole states in the ground state may be non-negligible if sufficiently strong interaction is applied. In a broader context, than the hereby considered, Hara [6] suggested to include explicitly the number of quasiparticles on each level, which turned out to have a dramatic effect on the collective properties of the low-lying states in open-shell even-even nuclei [15, 16]. Adapting this approach to the LMG model we can write

$$
\left\langle\left|n_{+1}\right|\right\rangle=N \rho,\left\langle\left|n_{-1}\right|\right\rangle=N(1-\rho) .
$$

Eq. (31) then transforms to

$$
\left\langle\left|s_{n}^{-}, s_{n^{\prime}}^{+}\right|\right\rangle=\delta_{n n^{\prime}}(1-2 \rho) .
$$

Analogous to Eq. (32) in the current context is the following one

$\left(\begin{array}{cc}A_{i i^{\prime}} & B_{i i^{\prime}} \\ B_{i i^{\prime}}^{*} & A_{i i^{\prime}}^{*}\end{array}\right)\left(\begin{array}{c}\psi_{i^{\prime}}^{(m)} \\ \varphi_{i^{\prime}}^{(m)}\end{array}\right)=\omega\left(\begin{array}{cc}U_{i i^{\prime}} & 0 \\ 0 & -U_{i i^{\prime}}^{*}\end{array}\right)\left(\begin{array}{c}\psi_{i^{\prime}}^{(m)} \\ \varphi_{i^{\prime}}^{(m)}\end{array}\right)$,

where

$$
\begin{gathered}
A_{n n^{\prime}}=G(1-2 \rho)^{2}-\delta_{n n^{\prime}}(1-2 \rho)(G-\varepsilon), \\
B_{n n^{\prime}}=G(1-2 \rho)\left(\delta_{n n^{\prime}}+2 \rho-1\right),
\end{gathered}
$$

and

$$
U_{n n^{\prime}}=\delta_{n n^{\prime}}(1-2 \rho)
$$

The solution of these equations is obtained to be

$$
\begin{aligned}
& \psi=\frac{1}{\sqrt{N(1-2 \rho)}} \frac{1+\chi / 2+\omega_{0} / \varepsilon}{\sqrt{\left(1+\frac{\omega_{0}}{\varepsilon}\right)\left(1+\chi+\frac{\omega_{0}}{\varepsilon}\right)}}, \\
& \varphi=\frac{1}{\sqrt{N(1-2 \rho)}} \frac{\chi / 2}{\sqrt{\left(1+\frac{\omega_{0}}{\varepsilon}\right)\left(1+\chi+\frac{\omega_{0}}{\varepsilon}\right)}},
\end{aligned}
$$

where

$$
\omega_{0}^{2}=\varepsilon^{2}(1+\chi), \quad \chi=\frac{2 G}{\varepsilon}[(1-2 \rho) N-1] .
$$

The system of equations closure is insured by the additional relation:

$$
\rho=\frac{(\varphi)^{2}}{1+2(\varphi)^{2}}
$$

In order to obtain the correct ERPA solution one needs to solve the system of coupled equations (45), (46), and (47).

\section{E. Phonon vacuum solution}

Finally, the featured method that we examine (conf. Sec. (II) translates in the language of the LMG model in the following way. The wave function (2) assumes the form

$$
\left.\left.|\rangle=N_{0} e^{\frac{1}{2} S \sum_{i i^{\prime}} s_{i}^{+} s_{i^{\prime}}^{+}}\right\rangle=N_{0} e^{\frac{1}{2} S J_{+}^{2}}\right\rangle .
$$

Up to arbitrary order $n \leq N / 2$ the ground-state energy is obtained to be

$$
\begin{aligned}
& \langle|H|\rangle=N_{0}^{2} \sum_{n} \frac{1}{(n !)^{2}}\left(\frac{S}{2}\right)^{2 n}\left\langle J_{-}^{2 n} H J_{+}^{2 n}\right\rangle+ \\
& 2 N_{0}^{2} \sum_{n} \frac{n}{(n !)^{2}}\left(\frac{S}{2}\right)^{2 n-1}\left\langle J_{-}^{2 n-2} H J_{+}^{2 n}\right\rangle
\end{aligned}
$$

with

$$
N_{0}^{2}=\left[\sum_{n} \frac{1}{(n !)^{2}}\left(\frac{S}{2}\right)^{2 n}\left\langle J_{-}^{2 n} J_{+}^{2 n}\right\rangle\right]^{-1}
$$

The variational equation $\partial_{S}\langle|H|\rangle=0$ then yields the following problem 


$$
\begin{aligned}
& N_{0}^{2} \sum_{n} \frac{n}{(n !)^{2}}\left(\frac{S}{2}\right)^{2 n-2}\left[\frac{S}{2}\left\langle J_{-}^{2 n} H J_{+}^{2 n}\right\rangle+(2 n-1)\left\langle J_{-}^{2 n-2} H J_{+}^{2 n}\right\rangle\right]+ \\
& \partial_{S} N_{0}^{2} \sum_{n} \frac{1}{(n !)^{2}}\left(\frac{S}{2}\right)^{2 n-1}\left[\frac{S}{2}\left\langle J_{-}^{2 n} H J_{+}^{2 n}\right\rangle+2 n\left\langle J_{-}^{2 n-2} H J_{+}^{2 n}\right\rangle\right]=0 .
\end{aligned}
$$

Respectively, the energy of the $1 p-1 h$ excited state is evaluated as

$$
\omega=\psi^{2}\left\langle\left|J_{-} H J_{+}\right|\right\rangle-2 \psi \varphi\left\langle\left|J_{-} H J_{-}\right|\right\rangle+\varphi^{2}\left\langle\left|J_{+} H J_{-}\right|\right\rangle
$$

The expressions for the Hamiltonian average values in Eqs.(51) and (52) are given in App. B The forward and backward phonon amplitudes in Eq.(52) are obtained by applying the normalization condition for the one-phonon state

$$
N_{0}^{2}\left(\psi^{2}-\varphi^{2}\right) \sum_{n}\left(\frac{1}{n !}\right)^{2}\left(\frac{S}{2}\right)^{2 n}(N-4 n)\left\langle J_{-}^{2 n} J_{+}^{2 n}\right\rangle=1,
$$

along with the definitive equation

$$
Q|\rangle=0,
$$

resulting in the relation

$$
[\varphi-(N-1) \psi S]^{2}+6 \psi^{2} S^{4}(N-1)(N-2)=0 .
$$

Note that the latter relation is independent of the expansion order $n$.

If we truncate the exponent expansion (48) to first order, i.e. allow for $2 p-2 h$ admixtures only in the groundstate wave function, we obtain

$$
N_{0}^{2}=\frac{1}{1+\frac{1}{2} N(N-1) S^{2}}
$$

The variational problem then is rewritten as

$$
\delta\left(N_{0}^{2}\left\langle\left(1+\frac{1}{2} S J_{-}^{2}\right) H\left(1+\frac{1}{2} S J_{+}^{2}\right)\right\rangle\right)=0 .
$$

The expressions for the relevant quantities in this equation are given in App. A. The structure coefficient $S$ in Eq.(48) is related to those in Eq.(3) in the following way:

$$
S=-\frac{4}{N} S_{0}(+1-1 ;+1-1) .
$$

Performing the variation (57) we get the following simple quadratic equation for $S$ :

$$
1+\left(2 \frac{\varepsilon}{G}+2 N-4\right) S-\frac{1}{2}\left(N^{2}-N\right) S^{2}=0 .
$$

The ground-state energy in this case evaluates to

$$
E=N_{0}^{2}\left[\langle H\rangle+N(N-1) G S+\frac{1}{4}(\varepsilon-G)(-N+4) N(N-1) S^{2}+G N(N-1)^{2} S^{2}\right] .
$$

The transition between the quantities obtained using the realistic Hamiltonian(14) and the LMG ones with
$2 p-2 h$ correlations only is performed in App $\mathrm{A}$ 

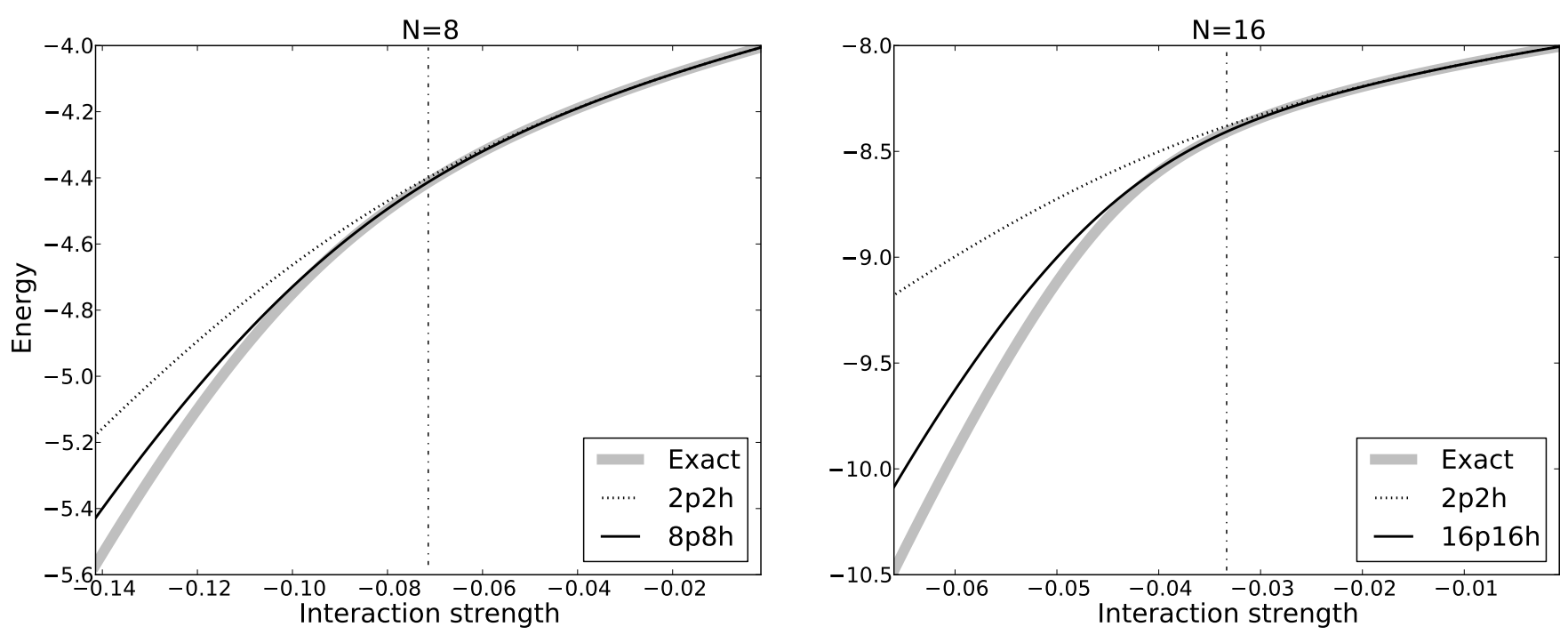

FIG. 1. Ground-state energy (MeV) in a two-level LMG model systems with $N=8$ (left panel) and $N=16$ (right panel) particles as function of the interaction strength $G(\mathrm{MeV})$. Solutions obtained using the phonon vacuum explicit variation with $2 p-2 h$ and up to $16 p-16 h$ admixtures into the ground-state wave function are compared to the exact one. The vertical dash-dotted line indicates the strength at which the RPA experiences a collapse. The energy gap $\varepsilon$ between the two LMG levels is set to $1 \mathrm{MeV}$.

\section{NUMERICAL RESULTS}

The three approximations, presented in the previous section, are compared with the exact solution based on the ground and first excited state energies (Fig 1) as well as based on the occupation particle density (Fig, 2) on the lower LMG model level in the ground state. We assigned $N \leq 16$ particles to the system for a simple reason - if we consider the two levels of the LMG model representing the valence sub-shells in the nucleus then these would be

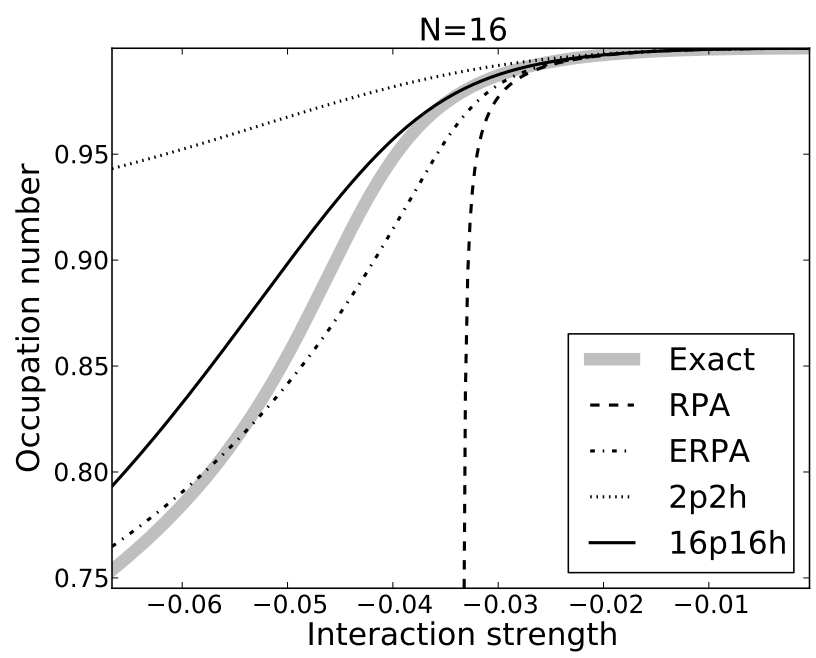

FIG. 2. Same as Fig. 1 but for the occupation number on the lower LMG model level for a system of $N=16$ particles. RPA and ERPA curves are also plotted. the source of the major effects for the low-lying states and $N=16$ would mimic the neutron or proton subsystem of a nucleus from the middle of $s d g h$ major shell.

The first observation in Fig. 1 is the clearly designated critical RPA strength

$$
G_{c r i t}=-\frac{\varepsilon}{2(N-1)}
$$

which separates the region where a real RPA solution can be found from the one, in which only a complex solution is obtained. It is also worthwhile to notice that the RPA point of collapse stands at the onset of the transition between the two nearly linear sections of the exact solution for the ground-state energy, which are more distinguished in systems with a larger number of particles.

On the other hand, the explicit variation of the phonon vacuum with $2 p-2 h$ admixtures only in the ground state yields solution at any $G$. However, as seen from Figs. 1 and 2, increasing the interaction strength beyond the RPA critical point causes progressive divergence of this solution from the exact one for both the ground-state energy and the occupation number. This divergence exacerbates incrementing the number of particles in the system. Adding higher order terms to the energy functional in Eq.(49), which account for further correlations effects, greatly improves the results bringing the energy of the phonon vacuum and the occupation number closer to the exact value. The importance of the multi-particle-hole admixtures to the ground state in the strong interaction regime is illustrated in Fig. 3] where the weight of the ground state's $0 p-0 h$ component is plotted as function of the interaction strength $G$. Dispite the reasonable agreement for the ground-state energies in this regime the wave 

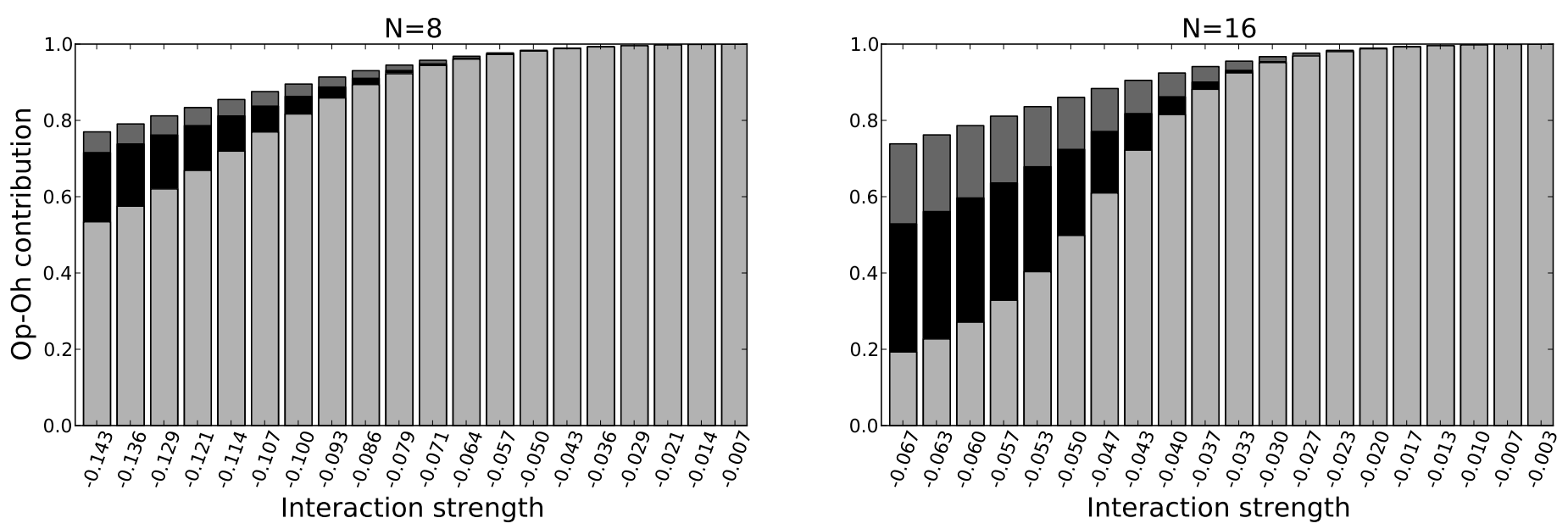

FIG. 3. The $0 p-0 h$ contribution to the exact ground-state wave function from Eq.(24) (light gray), the phonon vacuum solution from Eq. (48) with $2 p-2 h$ admixtures (dark gray) and with correlations of order $N / 2$ (black) as function of the interaction strength $G$ for LMG model systems with $N=8$ (left panel) and $N=16$ (right panel) particles.

function of the phonon vacuum yields a substantially less correlated state than the true ground state.The prescription of the variational principle for monotonic convergence is easily seen in Figs. 112, 3] and 4 as the higher order correlations bring the result closer to the exact solution but never overbinding the ground state.

The behaviour of the first excited state's energy within the phonon vacuum variational approach exhibits a collapse at interaction strengths far weaker than those at which the first excited state energy starts to diminish (see Fig (4). The position of this point is very much independent of the order of multi-particle-hole correlations included in the phonon vacuum state in cases when this point is near $G_{c r i t}$ (Eq. (61)) as in the weak interaction regime admixtures beyond the $2 p-2 h$ ones contribute relatively little (see Fig. 31). The obvious reason for this collapse, as noted in [18], can be attributed to the higher order particle-hole admixtures contributing in the structure of the first excited state which start to be an important factor as the interaction becomes stronger.

As opposed to the RPA, a real ERPA solution, is found everywhere in the range of $G$ values considered. In the interval $\left(G_{\text {crit }}, 0\right]$ it performs just as well as the RPA does except for strengths close to $G_{c r i t}$. In the strong interaction regime the ERPA gives rather good results both for the particle occupation and the first excited state energy (see Figs. 2 and (4). Near and beyond $G_{\text {crit }}$ it predicts higher depletion of the lower LMG level (see Fig 2) and correspondingly, as seen in Fig. 4, it gives lower value for the energy of the first excited state. At interactions twice as strong as the RPA point of collapse the first excited state's energy is found to be a bit higher compared to the exact solution. This result is to be expected given that the particle occupation number at such strengths within the ERPA is overestimated. It is worth noticing that in the strong interaction regime a solution of the system of coupled equations (45), (46) and (47) is found for an oc- cupation number whose value $\rho$ is in very close proximity to the critical occupation number

$$
\rho_{\text {crit }}=\frac{1}{2}-\frac{1}{2 N}\left(1-\frac{\varepsilon}{2 G}\right) .
$$

\section{CONCLUSION AND OUTLOOK}

In this work we initiated the development of a variational approach for approximating the ground state of nuclei using a wave function in the form of the phonon vacuum state, tailored to take into account the action of the long-range residual forces. Essentially it is an attempt to provide a controlled succession of approximations for estimating the contributions from different multi-particle-hole admixtures. Applying our idea to the very schematic LMG model, we showed the superiority of our approach over the RPA, based on comparison with the exact solution for the ground and first-excited state energies as well as based on the particle occupation numbers. Alongside that we adapted the ERPA [6] to the LMG model to show its high utility within a wide range of interaction strengths. As far as the LMG model is able to simulate real nuclei, we conclude the following:

- The RPA point of collapse separates two types of system's behavior - the weak and strong interaction regimes.

- The RPA provides an accurate and computationally efficient method to treat nuclei until the point of collapse inherent to this approximation.

- The ERPA improves over the standard RPA as it yields solution for any interaction strength. The calculated value for the first excited state's energy 

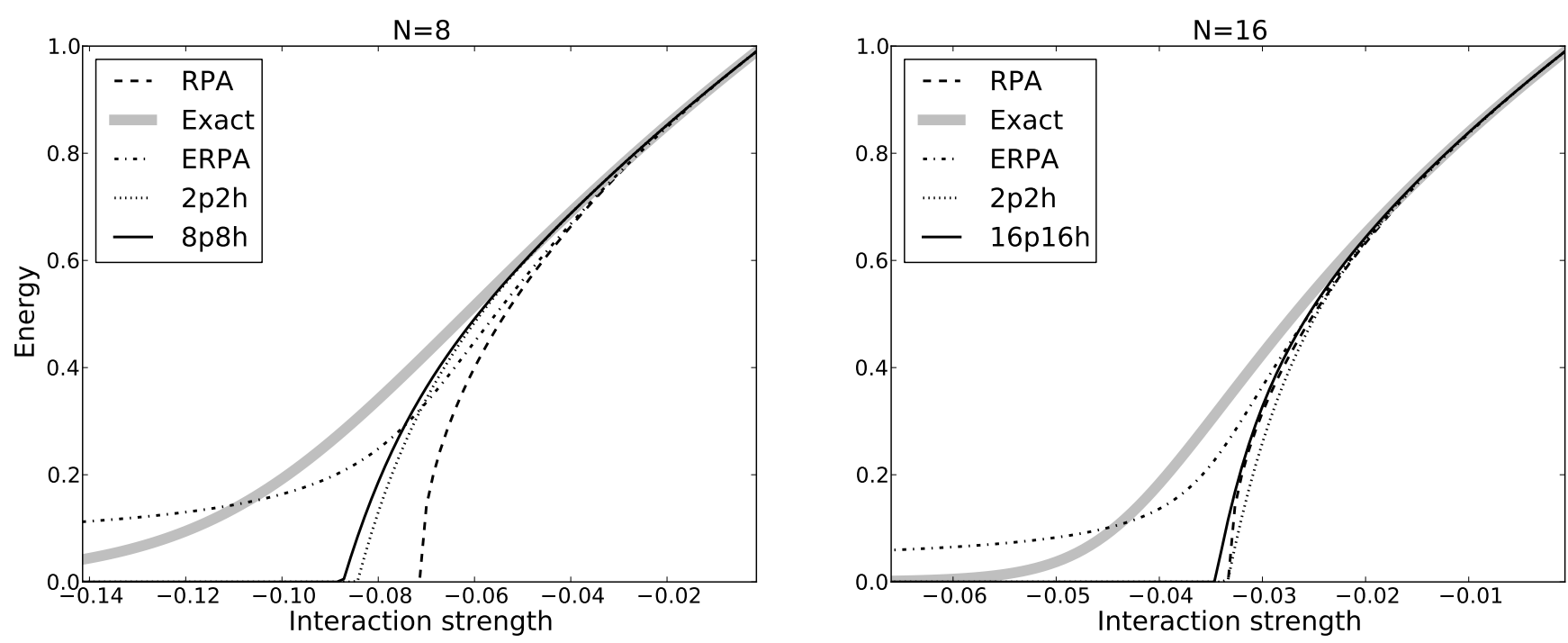

FIG. 4. Same as Fig. 1 but for the energy of the first excited state. RPA and ERPA curves are also plotted.

is the closest to the exact one amongst all considered approximations. The ground state occupation numbers within this approximation are also reproduced with reasonable accuracy.

- Explicitly varying the phonon vacuum state one obtains a very good accuracy to the ground-state energy as the result improves monotonically adding higher order correlations into the ground state. In the region beyond the RPA point of collapse, multiple particle-hole admixtures higher than $2 p-2 h$ ones start to play very important role and at such strengths, as seen from Fig. 3, the phonon vacuum solution significantly underestimates the degree of correlation. This effect is amplified with the increase of the number of particles in the system.

- The energy of the first excited state can be treated as an $1 p-1 h$ excitation over the ground state only in the weak interaction regime until the RPA point of collapse. Beyond that point it vanishes rapidly especially for systems with a larger number of particles and its proper description requires consideration within and extended configuration space for the excited states.

Applying the presented approach to real nuclei one can expect to obtain improved results than previously published for isotopes from the transitional regions.Being a many-body wave function this approach may be a good choice for describing phenomena involving many-particle correlations as, for example, cluster configurations. The influence of the long-range residual forces on the mean field and the pairing correlations in real nuclei is another perspective which the presented development makes possible to realize and it is currently progressing.

\section{ACKNOWLEDGMENTS}

The author is indebted to Prof. V.V. Voronov for helpful comments and valuable discussions during the course of this work. Thanks are also due to Prof. R.V. Jolos for his useful remarks.

This work is partially supported by JINR grant No. 13-302-07.

\section{Appendix A}

Here we give expressions for the quantities in Eq. (11) for single species of nucleons. A transition between these expressions and the ones obtained within the LMG model with $2 p-2 h$ correlations into the ground-state wave function is performed.

$$
\langle H\rangle=\sum_{12 \lambda} \kappa_{0}^{\lambda}\left(h_{12}^{\lambda}\right)^{2},
$$

$$
\begin{gathered}
\left\langle S^{\dagger} S\right\rangle=2 \sum_{1234 \lambda} \pi_{\lambda}^{2} S_{\lambda}^{2}(1234)+4 \sum_{1234 \lambda \lambda_{1}} \pi_{\lambda_{1}}^{2} \pi_{\lambda}^{2} S_{\lambda}(1234) S_{\lambda_{1}}(2431)\left\{\begin{array}{ccc}
3 & 4 & \lambda \\
2 & 1 & \lambda_{1}
\end{array}\right\}, \\
\langle H S\rangle=2 \sum_{1234 \lambda} \pi_{\lambda}^{2} \kappa_{0}^{\lambda} H_{\lambda}(1234) S_{\lambda}(1234)+4 \sum_{1234 \lambda \lambda_{1}} \pi_{\lambda_{1}}^{2} \pi_{\lambda}^{2} \kappa_{0}^{\lambda} H_{\lambda}(1234) S_{\lambda_{1}}(2431)\left\{\begin{array}{ccc}
3 & 4 & \lambda \\
2 & 1 & \lambda_{1}
\end{array}\right\},
\end{gathered}
$$




$$
\left\langle S^{\dagger} H S\right\rangle=\left\langle S^{\dagger} H_{1} S\right\rangle+\left\langle S^{\dagger} H_{2} S\right\rangle
$$

with

$$
\begin{gathered}
\left\langle S^{\dagger} H_{1} S\right\rangle=2 \sum_{1234 \lambda} \pi_{\lambda}^{2} \varepsilon_{1234} S_{\lambda}^{2}(1234)+4 \sum_{1234 \lambda \lambda_{1}} \pi_{\lambda_{1}}^{2} \pi_{\lambda}^{2} \varepsilon_{1234} S_{\lambda}(1234) S_{\lambda_{1}}(2431)\left\{\begin{array}{ccc}
3 & 4 & \lambda \\
2 & 1 & \lambda_{1}
\end{array}\right\} \\
\left\langle S^{\dagger} H_{2} S\right\rangle=-8 \sum_{1234 \lambda} \pi_{\lambda}^{2} \kappa_{0}^{\lambda} F_{\lambda}(1234) S_{\lambda_{1}}(1234)-16 \sum_{1234 \lambda \lambda_{1}} \pi_{\lambda}^{2} \pi_{\lambda_{1}}^{2} \kappa_{0}^{\lambda} F_{\lambda}(1234) S_{\lambda_{1}}(2431)\left\{\begin{array}{ccc}
3 & 4 & \lambda \\
2 & 1 & \lambda_{1}
\end{array}\right\} \\
+16 \sum_{1234 \lambda} \pi_{\lambda}^{2} R_{\lambda}(1234) S_{\lambda}(1234)+32 \sum_{1234 \lambda \lambda_{1}} \pi_{\lambda}^{2} \pi_{\lambda_{1}}^{2} R_{\lambda}(1234) S_{\lambda_{1}}(2431)\left\{\begin{array}{ccc}
3 & 4 & \lambda \\
2 & 1 & \lambda_{1}
\end{array}\right\} \\
-\left(\sum_{a a^{\prime} J}\left(h_{a a^{\prime}}^{J}\right)^{2} \kappa_{0}^{J}\right)\left[2 \sum_{1234 \lambda} \pi_{\lambda}^{2} S_{\lambda}^{2}(1234)+4 \sum_{1234 \lambda \lambda_{1}} \pi_{\lambda}^{2} \pi_{\lambda_{1}}^{2} S_{\lambda}(1234) S_{\lambda_{1}}(2431)\left\{\begin{array}{ccc}
3 & 4 & \lambda \\
2 & 1 & \lambda_{1}
\end{array}\right\}\right],
\end{gathered}
$$

where

$$
F_{\lambda}(1234)=\frac{h_{34}^{\lambda}}{\pi_{\lambda}^{2}} \sum_{56} h_{56}^{\lambda}\left[S_{\lambda}(1256)+2 \sum_{J} \pi_{J}^{2}\left\{\begin{array}{lll}
5 & 6 & \lambda \\
1 & 2 & J
\end{array}\right\} S_{J}(5216)\right]
$$

and

$$
R_{\lambda}(1234)=\left(\sum_{5 J} \kappa_{0}^{J}\left(h_{15}^{J}\right)^{2}\right) \frac{S_{\lambda}(1234)}{\pi_{33}}
$$

Here we used the shortcut notation

$$
H_{\lambda}(1234)=h_{12}^{\lambda} h_{34}^{\lambda}
$$

where

$$
\begin{aligned}
& \left\langle S^{\dagger} S\right\rangle=\frac{1}{2} N(N-1) S^{2}, \\
& \langle H S\rangle=\frac{1}{2} G N(N-1) S,
\end{aligned}
$$

$$
h_{12}^{\lambda}=\frac{f_{12}^{\lambda} u_{12}^{+}}{2}
$$

$$
\left\langle S^{\dagger} H S\right\rangle=\left[\frac{(\varepsilon-G)(4-N) N(N-1)}{4}+G N(N-1)^{2}\right] S^{2} .
$$

In Eq. A5 $\varepsilon_{1234}$ stands for the energy of a fourquasiparticle state, i.e. $\varepsilon_{1234}=\varepsilon_{1}+\varepsilon_{2}+\varepsilon_{3}+\varepsilon_{4}$. In Eq.(A4) the term in the Hamiltonian (14), quadratic with respect to the operator $B$, defined in Eq.(5), is omitted.

Applying the considerations from Sec IIIA and using the relation

$$
\left\{\begin{array}{lll}
j & j & 0 \\
j & j & 0
\end{array}\right\}=-\frac{1}{2 j+1}=-\frac{1}{N}
$$

one obtains the corresponding LMG model expressions with $2 p-2 h$ correlations in the ground-state wave function:

$$
\langle H\rangle=(G-\varepsilon) \frac{N}{2}
$$

\section{Appendix B}

In this Appendix we give the expressions for the Hamiltonian average values in the uncorrelated $\rangle$ and correlated |) ground states needed to solve Eqs. (51), (53) and (55) from Sec. IIIE.

$$
\begin{gathered}
\left\langle J_{-}^{n} J_{+}^{n}\right\rangle=\frac{n ! N !}{(N-n) !} \\
\left\langle J_{-}^{n} H J_{+}^{n+2}\right\rangle=\frac{G}{2} \frac{(n+2) ! N !}{(N-(n+2)) !} \\
\left\langle J_{-}^{n} H J_{+}^{n}\right\rangle=\left[(\varepsilon+G)\left(n-\frac{N}{2}\right)+G(n+1)(N-n)\right] \frac{n ! N !}{(N-n) !}
\end{gathered}
$$




$$
\begin{gathered}
\left\langle\left|J_{-} H J_{+}\right|\right\rangle=N_{0}^{2} \sum_{n} \frac{1}{(n !)^{2}}\left(\frac{S}{2}\right)^{2 n}\left\langle J_{-}^{2 n+1} H J_{+}^{2 n+1}\right\rangle+2 N_{0}^{2} \sum_{n} \frac{n}{(n !)^{2}}\left(\frac{S}{2}\right)^{2 n-1}\left\langle J_{-}^{2 n-1} H J_{+}^{2 n+1}\right\rangle \\
\left\langle\left|J_{-} H J_{-}\right|\right\rangle=N_{0}^{2} \sum_{n} \frac{1}{(n !)^{2}} 2 n(N-2 n+1)\left(\frac{S}{2}\right)^{2 n}\left\langle J_{-}^{2 n+1} H J_{+}^{2 n-1}\right\rangle+N_{0}^{2} \sum_{n} \frac{n}{(n !)^{2}}\left(\frac{S}{2}\right)^{2 n-1} 2 n(N-2 n+1)\left\langle J_{-}^{2 n-1} H J_{+}^{2 n-1}\right\rangle \\
(\mathrm{B} 5) \\
\left\langle\left|J_{+} H J_{-}\right|\right\rangle=N_{0}^{2} \sum_{n} \frac{1}{(n !)^{2}} 4 n^{2}(N-2 n+1)^{2}\left(\frac{S}{2}\right)^{2 n}\left\langle J_{-}^{2 n-1} H J_{+}^{2 n-1}\right\rangle+ \\
N_{0}^{2} \sum_{n} \frac{n}{(n !)^{2}}\left(\frac{S}{2}\right)^{2 n-1}(2 n-2) 2 n(N-2 n+3)(N-2 n+1)\left\langle J_{-}^{2 n-3} H J_{+}^{2 n-1}\right\rangle
\end{gathered}
$$

[1] M. Baranger, Phys. Rev. 120, 957 (1960).

[2] K. Sawada, Phys. Rev. 106, 372 (1957).

[3] M. Bender, G.F. Bertsch, and P.-H. Heenen, Phys. Rev. C 73, 034322 (2006).

[4] D. Rowe, Nuclear collective motion. London: Menthuen, 1970.

[5] C. Qi, R. Liotta, and R. Wyss, Jour. of Phys: Conf. Series 338, 012027 (2012).

[6] K. Hara, Progr. Theor. Phys. 32, 88 (1964).

[7] V. G. Soloviev, Theory of Atomic Nuclei: Quasiparticles and Phonons (Institute of Physics, Bristol and Philadelphia, 1992).

[8] R.A. Sorensen, Nucl. Phys. 25, 674 (1961).

[9] D. Rowe, Proceedings of Frontiers of Collective Motions, pp. 220-229 (2003).

[10] A. Goswami, M.K. Pal, Nucl. Phys. 44, 294 (1963).

[11] J. Bardeen, L. N. Cooper, and J. R. Schrieffer, Phys. Rev. 108, 1175 (1957).

[12] E.A. Sanderson, Phys Lett. 19(2), 141 (1965).

[13] G. Brown, G. Jacob, Nucl. Phys. 42, 177 (1963).

[14] R. V. Jolos, W. Rybarska, Z. Phys. A 296, 73 (1980).

[15] D. Karadjov, V.V. Voronov, and F. Catara, Phys. Lett. B 306, 197 (1993); V.V. Voronov, D. Karadjov, F. Catara and A.P. Severyukhin, Phys. Part. Nucl., 31(4) (2000) 452 .
[16] S. Mishev and V.V. Voronov, Phys. Rev. C 82, 064312 (2010).

[17] P. Schuck, S. Ethofer, Nucl. Phys. A212(2) (1973) 269; J. Dukelsky, P. Schuck, Nucl. Phys. A512(3), 466 (1990).

[18] J.G. Hirsch, O. Civitarese, and M. Reboiro, Phys. Rev. C 60, 024309 (1999).

[19] K. Hagino, G.F. Bertsch, Phys. Rev. C 61 (2000) 024307, Nucl. Phys. A679, 1632000.

[20] I. Stetcu, C.W. Johnson, Phys. Rev. C 66 (2002) 034301.

[21] D.J. Rowe, 1968 Rev. Mod. Phys. 40153

[22] H. Lenske and J. Wambach, Phys. Lett. B 249, 377 (1990)

[23] F. Catara, N.D. Dang and M. Sambataro, Nucl. Phys. A 579 (1994) 1.

[24] F. Catara, G. Piccitto, M. Sambataro, and N. Van Giai, Phys. Rev. B 54, 17536 (1996)

[25] M. Grasso and F. Catara, Phys. Rev. C 63, 014317 (2000)

[26] A.A Kuliev, A. Faessler, M. Guner, and V. Rodin, Jour. of Phys. G, 30(9), 1253 (2004).

[27] D.S. Delion, P. Schuck, and J. Dukelsky, Phys. Rev. C 72, 064305 (2005).

[28] M. Jemai and P. Schuck, Phys. Atom. Nucl. 74(8), 1139 (2011).

[29] H. Lipkin, N. Meshkov, A. Glick, Nucl. Phys. 62, 188 (1965).

[30] J.A Maruhn, P.-G. Reinhard, and E. Suraud, Simple Models of Many-Fermion systems (Springer, 2010).

[31] P. Ring and P. Schuck, The Nuclear Many-Body Problem (Springer-Verlag, New York, 1980). 